Memoirs of the College of Science, University of Kyoto, Series A

Vol. XXXI, Mathematics No. 1, 1958.

\title{
Some Basic Theorems in Partial Differential Algebra
}

\author{
By \\ A. SEIDENBERG \\ (Communicated by Prof. Akizuki, September 1, 1957)
}

Introduction. Let $F$ be an arbitrary partial differential field of characteristic 0 with $m$ types of differentiation $\delta_{1}, \cdots, \delta_{m}, m \geq 0$, and let $u$ be an element from an extension field $K$ of $F$. In 1934, Raudenbush [4] defined $u$ to be algebraic over $F$ if $n$ together with its derivatives satisfy some non-trivial polynomial relation over $F$. There are, of course, the three well-known to verify:

Axiom 1. $u_{i}$ is algebraic over $F\left\langle u_{1}, \cdots, u_{n}\right\rangle, i=1, \cdots, n$.

Axiom 2. If $u$ is algebraic over $F\langle v\rangle$ but not over $F$, then $v$ is algebraic over $F\langle u\rangle$.

Axiom 3. If $v$ is algebraic over $F\left\langle u_{1}, \cdots, u_{n}\right\rangle$ and each $u_{i}$ is algebraic over $F$, then $v$ is algebraic over $F$.

Axiom 1 is trivial; and Axiom 2 also follows easily. Axiom 3, however, required a straightforward but rather complicated computational argument. The Steinitz theory of transcendency was thus established. One may say that Raudenbush's definition (to be referred to as Definition I) is adapted to Axiom 2, but not to Axiom 3. On the other hand, consider the following definition. By the $\delta_{i}$-theory we mean the theory which results from regarding $K$ as a partial differential field under the $m-1$ differentiations $\delta_{1}, \cdots, \delta_{i-1}, \delta_{i+1}, \cdots, \delta_{m}$. The definition runs :

Definition. 1I. We say that $u$ is algebric over $F($ for $m>0)$ if $F\langle u\rangle / F$ is of finite degree of transcendency in each of the $\delta_{i^{-}}$ theories, $i=1, \cdots, m$. (For $m=0$, the usual definition is to obtain.) Here Axiom 3, stated in terms of Definition II, becomes :

If $\left.\left.F<u_{1}, \cdots, u_{n}, v\right\rangle / F<u_{1}, \cdots, u_{n}\right\rangle$ and $F<u_{1}, \cdots, u_{n}>/ F$ are both of finite degree of transcendency in each of the $\delta_{i}$-theories, 
then $F\langle v\rangle / F$ is also of finite degree of transcendency in each of the $\delta_{i}$-theories.

This, however, is (or would be) a standard fact on degrees of transcendency for fields with $m-1$ types of differentiation. Axiom 3 may thus be regarded as established inductively, This time though, Axiom 2 gives difficulty. If, however, one includes the assertion that the two definitions are equivalent as a theorem in an inductive scheme, then Axioms 2 and 3 are, as we have just noted, immediate; and the equivalence of the two definitions also follows at once by induction. Thus the theory of transcendency is established in a few lines and without the examination of computational details.

The question now is whether a similar situation obtains for characteristic $p>0$. Here we may remark that it is not off-hand easy to say what the definition should be. Definitions I and II are out as they fail already for $m=1$ : for counter-examples see [5; pp. 182-183]. Observing that in the case $m=1, \quad p=0$ the words "finite degree of transcendency" could be equivalently replaced by the word "finite" in Definition II, Seidenberg [5] took this modified definition as the basis for his theory with $m=1$, $p>0$. The modified definition fails, however, even for $p=0$ if $m>1$. Thus we seem to be at an impasse.

Taking up the case $m>0, p>0$, Okugawa [3] gave a good definition of algebraic dependence. Let $U$ be an indeterminate and $G(U)$ an element of the polynomial ring $F\{U\}$. Let $X$ be a derivative of $U$. Then $G$ can be written uniquely in the form $G=A_{0}+A_{1} X+\cdots+A_{p-1} X^{p-1}$, where the $A_{i}$ are elements of $F\{U\}$ involving $X$ only to powers divisible by $p$. Okugawa then says that $u$ is algebraic over $F$ if there is some polynomial $G$, written in the above form, for which $G(u)=0$ and $A_{i}(u) \neq 0$ for some $i>0$. An equivalent formulation is as follows: let the $p^{t h}$ powers of $u$ and its derivatives be adjoined to $F$ to give the differential field $F_{1}$; then Definition I is to obtain relative to $F_{1}$ with the additional requirement that the polynomial in question be of degree at most $p-1$ in any of the derivatives of $U$. Since this definition comes by modifying Raudenbush's, we refer to it as Definition I'.

Okugawa dismisses the verification of the axioms with the assertion that they "can be proved by modifying the method of Raudenbush". This is undoubtedly so. Passing on to some of 
Okugawa's applications of his theory of transcendence, one comes to the the theorem of MacLane on separating transcendency bases. Here in the proof (p. 105) one reads: "As $F\left\langle x_{1}, \cdots, x_{n}\right\rangle$ is separable over $F$ and $F\left\langle x_{k_{1}}, \cdots, x_{k_{r}}\right\rangle$ is purely transcendental over $F, F\left\langle x_{1}, \cdots, x_{n}\right\rangle$ is also separable over $F\left\langle x_{k_{1}}, \cdots, x_{k_{r}}\right\rangle$ ". The argument here seems faulty; it seems to be suggested that if $K, L, M$ are three fields with $K \subseteq L \subseteq M, M / K$ is separable, and $L$ a pure transcendental extension of $K$, then $M / L$ is separable. This is certainly false in the abstract case as one sees by taking $L=K\left(x^{p}\right), M=K(x)$. The argument is not saved by the fact that the assertion does obtain in the differential situation $(m>0)$, as we shall show below, because while the assertion is true, it is much stronger than the theorem Okugawa was proving and nowise follows from the context. We also call attention to the statement earlier on page 105 that "if $u_{i}$ is the $x$-derivative corresponding to $U_{i}$, then $F\left\langle x_{1}, \cdots, x_{n}\right\rangle=F\left(x^{(\tau)}\right)\left(u_{1}, \cdots, u_{t}\right)$ and $F\left\langle x_{1}, \cdots, x_{n}\right\rangle$ is separable algebraic over $F\left(x^{(\tau)}\right)$ ". Here Okugawa appears to be overlooking the fact that the relation which relates $u_{1}$, say, to the variables $x^{(\tau)}$ also may involve proper derivatives of $u_{1}$. In any event, we are unable to decide the status of the statement. If it is true, it is harder to prove than the theorem itself; and if it is false, it equally is hard to give a counter-example. Although the theorems dealt with below are elementary, they are basic, and in view of the lacunae in Okugawa's paper, ${ }^{13}$ it is perhaps of value to take up these points once more, especially as the proofs for $p>0$ can be made just as neat as for $p=0$. At the same time we consider the theorem of the primitive element and the theorem on polynomial inequalities and show that these theorems are equivalent, a result new also in case $p=0$; and we extend MacLane's theorem for $m>1$ just as was done in [6] for $m=1$.

1) In this connection see also [2]. Incidentally, in this review Kolchin says that Okugawa's definition of algebraic dependence is "in the ordinary case, stronger" than Seidenberg's. Also Okugawa, in footnote 6, says that one of the definitions implies the other, but he does not say that the definitions are equivalent. It is possible from these statements to get the impression that Okugawa's definition supercedes Seidenberg's. Such impression would be quite false, as the two definitions are equivalent. In [5], by the way, the definition was not simply put down ad hoc, but by an analysis it was indicated why it was the only definition likely to be fruitful. Okugawa's work does not contradict this expectation, but, on the contrary, substantiates it. 
1. The theory of transcendence. We take Okugawa's definition (Definition $\mathrm{I}^{\prime}$ ) as being adapted to Axiom 2. What shall be the definition adapted to Axiom 3? Strangely enough, it will be Definition II as given above! It is true that the definition fails for $m=1$, but it is correct for $m>1$, and our induction will start from $m=1$. (In the case of $p=0$, one can start from $m=0$; not so for $p>0$.) For $m=1$, Definition II is to be modified as in [5], that is, the words "finite degree of transcendence" are to be replaced by the word "finite". To be quite explicit, we put down the definition again, since it does deviate slightly from II.

Definition II'. For $m=1$, we say that $u$ is algebraic over $F$ if $F\langle u\rangle / F$ is finite. For $m>1$, we say that $u$ is algebraic over $F$ if $F\langle u\rangle / F$ is of finite degree of transcendency in each of the $\delta_{i}$-theories, $i=1, \cdots, m$.

Postponing consideration of the case $m=1$, we proceed to the inductive part of the proof ${ }^{2}$. Here the argument is parallel to the argument given for $p=0$ with one additional point. The additional point is as follows: In the various arguments involving variables $u, v, \cdots$ over $F$ we adjoin the $p^{t h}$ powers of $u, v$, and their derivatives to obtain the field $F_{1}$, the polynomials in question are then restricted to be of degree at most $p-1$ in any of the (algebraically indeterminate) letters, a conclusion is drawn relative to $F_{1}$ and then the $p^{t h}$ powers are eliminated from $F_{1}$, by means of the following lemma, to give the desired conclusion relative to $F$.

Lemma 1. Let $K, L$ be differential fields $(m \geq 1), K \subseteq L$, and assume that $L$ is obtained from $K$ by the adjunction of a finite or infinite set of constants. Then if $u$ is algebraic over $L$, it is also algebraic over $K$.

Proof. It is understood that this lemma is part of an inductive schems (which includes the statement that Definitions $\mathrm{I}^{\prime}$ and $\mathrm{II}^{\prime}$ are equivalent and satisfy the axioms (as well as the standard theory of transcendence which follows from them)). We proceed to verify the induction. Suppose that $L$ is obtained from $K$ by the adjunction of a finite set of constants. We have that $L\langle u\rangle / L$

2) For $m=1$, Definition $\mathrm{II}^{\prime}$ coincides with the definition of [5], where the axioms were checked. The equivalence of the two definitions for $m=1$ is also immediate. Thus the first stage of the induction is established. The argument of the inductive part, however, gives this result anew and in a simpler way. 
is of finite degree of transcendence in each of the $\delta_{i}$-theories. Since $L / K$ is of finite degree of transcendence (in fact, even finite), also $L\langle u\rangle \mid K$ and $K\langle u\rangle / K$ are of finite degree of transcendence in each of the $\delta_{i}$-theories. Hence $u$ is algebraic over $K$. Since it requires only a finite number of elements from $L$ to bring the algebraic dependence of $u$ on $L$ to expression, we see that the finiteness condition on $L$ can be removed. The proof for $m=1$ is quite the same. This completes the proof of theorem, pending the verification of the other parts of the induction.

To complete the induction, little has to be added. The verification of Axiom 3 is quite immediate, using Definition II'. In Axiom 2, using Definition $I^{\prime}$, there is one slight new point: the relation relating $v$ to $u$ over $F$ must involve $u$, to be sure, but one has to contend with the possibility that $u$ and its derivatives occur only to powers divisible by $p$-Lemma 1 , however, disposes of this possibility at once. Thus the Axioms are verified. As to the equivalence of the definitions, the condition of Definition $I^{\prime}$ follows at once from the condition of Definition $\mathrm{II}^{\prime}$, even without the intervention of Lemma 1 (on the $m^{\text {th }}$ level); and the condition of Definition $\mathrm{II}^{\prime}$ follows from that of $\mathrm{I}^{\prime}$ : here use is made of Lemma 1 but there is no other difficulty. Thus the inductive part of the argument is completed.

Finally there is the case $m=1$ : but by now there is no new point, and further remarks may be omitted for the sake of brevity. Thus we have proved the following theorem.

Theorem 1. Definitions $\mathrm{I}^{\prime}$ and $\mathrm{II}^{\prime}$ are equivalent and satisfy the axioms. Hence the Steinitz transcendency theory also holds for partial differential algebra of arbitrary characteristic.

Remark. Note that if the condition of Definition $\mathrm{II}^{\prime}$ holds for one $i$, then the condition of Definition $\mathrm{I}^{\prime}$ holds, whence the condition of Definition $\mathrm{II}^{\prime}$ holds for all $i, i=1, \cdots, m$.

2. Separating transcendency bases. We shall need the following lemma.

Lemma 2. If $u_{1}, \cdots, u_{q}$ are algebraically independent over $F$ and $F\left\langle u_{1}, \cdots, u_{q}\right\rangle \mid F$ is separable, then the derivatives of the $u_{i}$ are algebraically independent (in the algebraic sense) over $F$.

The proof is immediate using Definition $I^{\prime}$ and the fact that elements in $F\left\langle u_{1}, \cdots, u_{q}\right\rangle$ linearly independent over $F$ are still such over $F^{1 / p}$. 
Theorem 2. Let $K, L, \Sigma$ be differential fields with $m$ types of differentiation, $m>0, K \subseteq L \subseteq \Sigma$. If $\Sigma / K$ is separable and $L / K$ is pure transcendental (in the differetial sense), then $\Sigma / L$ is separable.

Proof. We must show that elements $\sigma_{1}, \cdots, \sigma_{k} \in \Sigma$ which are linearly independent over $L$ remain such over $L^{1 / p}$, or that if $\sigma_{1}^{p}, \cdots, \sigma_{k}^{p}$ are linearly dependent over $L$, so are $\sigma_{1}, \cdots, \sigma_{k}$. We have $L=K\left\langle u_{1}, \cdots\right\rangle$, where the $u_{i}$ are algebraically independent over $K$, and since $L / K$ is separable, these $u_{i}$ and their derivatives are algebraically independent (in the algebraic sense) over $K$. We have $l_{1}, \cdots, l_{k} \in L$ not all zero such that $\sum l_{i} \sigma_{i}^{n}=0$. The $l_{i}$ may be supposed to be in $K\left\{u_{1}, \cdots\right\}$. Let $X_{1}, \cdots$ be indeterminates over $\Sigma$ and $l_{i}(X)$ polynomials over $K$ such that $l_{i}(u)=l_{i}$. We suppose $G(X)=\sum l_{i}(X) \sigma_{i}^{p}=1=0$, as $G(X)=0$ implies that the $\sigma_{i}^{p}$, hence also the $\sigma_{i}$, are linearly dependent over $K$; also we suppose $G(X)$ to be of minimal degree. Let $\pi_{1}, \pi_{2}, \cdots$ be the power-products in one of the $X_{i}$ and its derivatives $X_{i j}$ with degree at most $p-1$ in any letter; say $X_{i}=X_{1}$. Rewrite $G(X)$ as a linear combination of the $\pi_{i}$ with coefficients in $K\left\{\sigma_{1}^{p}, \cdots, \sigma_{k}^{p} ; X_{2}, \cdots ; \cdots, X_{1 j}^{p}, \cdots\right\}$. These coefficients must vanish for $X_{i}=u_{i}$, as otherwise one finds that $u_{1}$ is algebraic over $K\left\langle\sigma_{1}^{p}, \cdots, \sigma_{k}^{n} ; u_{2}, \cdots\right\rangle$, hence over $K\left\langle u_{2}, \cdots\right\rangle$, but this is not so. Because $G(X)$ is of minimal degree, this shows that the $X_{1 j}$, hence also the $X_{i j}$, occur in $G(X)$ with exponents divisible by $p$. Hence $l_{1}(X)=\sum c_{i j} m_{j}^{p}$, where the $m_{j}$ are powerproducts of the $X^{\prime} s$ and their derivatives, the $c_{i j} \in K$, not all $c_{i j}=0$. Hence $\sum c_{i j}\left(m_{j}(u) \sigma_{i}\right)^{p}=0$. By the separability of $\Sigma / K$ we have $\sum d_{i j} m_{j}(u) \sigma_{i}=0, d_{i j} \in K$, not all $d_{i j}=0$. Since the $u_{i j}$ are algebraically independent over $K$, also $\sum d_{i j} m_{j}(u) \neq 0$ for at least one $i$; whence the $\sigma_{i}$ are linearly dependent over $L$. This completes the proof.

3. The theorem on polynomial inequalities. Given a polynomial $G \neq 0$ in $F\left\{U_{1}, \cdots, U_{q}\right\}$, the question is whether elements $u_{i} \in F$ can be chosen so that $G(u) \neq 0$. The case $p=0, m \geq 1$ has been considered in [1], where it is shown that the theorem holds if and only if there exist $m$ elements $u_{i}$ in $F$ for which the Jacobian does not vanish. By an easy reformulation this is so if and only if $\delta_{1}, \cdots, \delta_{m}$ are linearly independent over $F$. The case $m=1, p>0$ has been dealt with in [5]; the case $p>0, m>1$, however, involves a new difficulty. In the proof in [1], Kolchin 
uses a device which typically will not work for $p>0$; a perusal of his proof gives, however, the following information ${ }^{3}$ :

The $\delta_{i}$ being linearly independent, if $G$ contains a term in which each $\delta_{i}$ occurs to at most the order $p--1$, then there exist elements $u \in F$ such that $G(u) \neq 0$.

We will now go on to give a criterion for an arbitrary polynomial. From the Leibniz formula for $\delta^{n}(u v)$ we see that $\delta^{p}(u v)=u \delta^{p} v+v \delta^{p} u$; in other words $\delta^{p}$ is, along with $\delta$, a derivation. We see then that $F$ is also a differential field under the derivations $\delta_{1}, \cdots, \delta_{m} ; \delta_{1}^{p}, \cdots, \delta_{m}^{p}$; etc. Moreover any polynomial $G$ under $\delta_{1}, \cdots, \delta_{m}$ can be rewritten equivalently as a polynomial under $\delta_{1}, \cdots, \delta_{m} ; \delta_{1}^{n}, \cdots, \delta_{m}^{n}$; etc. such that each term is of at most the order $p-1$ in any derivation. Thus we obtain the following theorem.

Theorem 3. The theorem on polynomial inequalities holds over $F$ if and only if $\delta_{1}, \cdots, \delta_{m} ; \delta_{1}^{p}, \cdots, \delta_{m}^{p}$; etc. are linearly independent over $F$.

4. The theorem of the primitive element. Let $F$ be a partial differential field, $u, v$ elements in an extension field of $F$ and algebraic over $F ; \Lambda$, an indeterminate over $F\langle u, v\rangle$. As Okugawa [3] observes, a computation like that in $[5 ;$ p. 176] shows that $F\langle\Lambda\rangle\langle u, v\rangle=F\langle\Lambda\rangle\langle u+\Lambda v\rangle$. By the parenthetical remark in [5; p. 177], one sees then that the theorem of the primitive element holds over $F$ if the theorem on polynomial inequalities holds over $F$.

The theorem on polynomial inequalities thus plays an essential role in the proof of the theorem of the primitive element, but its necessity has not hitherto been considered. In fact, however, it is necessary. First, a lemma. In order to have a notation uniform for all $p$, we designate $\delta_{1}^{n}, \cdots$ as $\delta_{m+1}, \cdots$.

Lemma. If $\delta_{1}, \cdots, \delta_{s}$ are linearly dependent over $F$, then they are also linearly dependent over $F_{0}$, the constant-field of $F$.

Proof. Let $a_{i_{1}} \delta_{i_{1}}+\cdots+a_{i_{k}} \delta_{i_{k}}=0, a_{i_{j}} \in F, \Pi a_{i_{j}} \neq 0, k$ minimal. Assume without loss of generality that $a_{i_{1}}=1$; then $a_{i_{2}}, \cdots, a_{i_{k}}$ are constants. In fact for any of the derivations $\delta$ we have:

3) One will need that the constant-field $F_{0}$ is infinite; but this is so. See [5; p. 185]. 


$$
\begin{aligned}
0 & =\delta\left(a_{i_{1}} \delta_{i_{1}}+\cdots+a_{i_{k}} \delta_{i_{k}}\right)=\left(\delta a_{i_{1}}\right) \delta_{i_{1}}+\cdots+\left(\delta a_{i_{k}}\right) \delta_{i_{k}}+\left(a_{i_{1}} \delta_{i_{1}}+\cdots+a_{i_{k}} \delta_{i_{k}}\right) \delta \\
& =\left(\delta a_{i_{2}}\right) \delta_{i_{2}}+\cdots+\left(\delta a_{i_{k}}\right) \delta_{i_{k}},
\end{aligned}
$$

whence $\delta a_{i_{2}}=\cdots=\delta a_{i_{k}}=0$ by the minimal propery of $k$, Q. E. D.

Theorem 4. The theorem of the primitive element holds over $F$ if and only if the theorem on polynomial inequalities holds over F. $\quad($ Here $p \geq 0, m \geq 1$.)

Proof. We give the proof for $p=0$; a similar but notationally slightly more complicated proof holds for $p>0$.

Assume, then that $\delta_{1}, \cdots, \delta_{m}$ are linearly dependent over $F$, hence also over $F_{0}$, so that $D \equiv c_{1} \delta_{1}+\cdots+c_{m} \delta_{m}=0$ for some $c_{i} \in F_{0}$, not all $c_{i}=0$. Say $c_{m} \neq 0$; then any solution of $D U=0$ has in the $\delta_{m}$-theory a degree of transcendency 1 . Let $u$ be one such; then one verifies that every element of $F\langle u\rangle$ satisfies $D U=0$ : in particular $D \delta_{i} u=0$ because the $c_{i}$ are constants. So the $\delta_{i}$ are still linearly dependent over $F\langle u\rangle$. Similarly we adjoin another solution $v$ of $D U=0$ so that the $\delta_{m}$-degree of transcendency of $F\langle u, v\rangle / F$ is 2. Then $F\langle u, v\rangle=F\langle w\rangle$ is clearly impossible, since the $\delta_{m}$-degree of trancendency of $F<w>/ F \leq 1$. This completes the proof.

\section{University of California \\ Berkeley 4, California}

\section{REFERENCES}

[1] E. R. Kolchin, Extensions of differential fields, Annals of Math., vol. 43 (1942), pp. 724-729.

[2] E. R. Kolchin, Review of Okugawa's paper [3], Math. Reviews, vol. 16, no. 6 (1955), p. $562 \mathrm{f}$.

[3] K. Okugawa, On diff erential algebra of arbitrary characteristic, Mem. of the College of Sci., Univer. of Kyoto, Ser. A, vol 28 (1953), pp. 97-107.

[4] H.W. Raudenbush, Hypertranscendental adjunctions to partial differential fields, Bull. Amer. Math. Soc., vol. 40 (1934), pp. 714-720.

[5] A. Seidenberg, Some basic theorems in differential algebra (characteristic p, arbitrary), Trans. Amer. Math. Soc., vol. 73 (1952), pp. 174-190.

[6] A. Seidenberg, On separating transcendency bases for differential fields, Proc. Amer. Math. Soc., vol. 6 (1955), pp. 726-728. 